\title{
Incremental value of diastolic stress test in identifying subclinical heart failure in patients with diabetes mellitus
}

\author{
Tomoko Nishi ${ }^{1,2 \dagger}$, Yukari Kobayashi (1) ${ }^{1,2 \dagger *}$, Jeffrey W. Christle ${ }^{1,3}$, Nicholas \\ Cauwenberghs $^{4}$, Kalyani Boralkar ${ }^{1,2}$, Kegan Moneghetti ${ }^{1,2,3}$, Myriam Amsallem ${ }^{1,2}$, \\ Kristofer Hedman ${ }^{2,5,6}$, KévinContrepois ${ }^{7}$, Jonathan Myers ${ }^{8}$, Kenneth W. Mahaffey ${ }^{9}$, \\ Ingela Schnittger ${ }^{1,2}$, Tatiana Kuznetsova ${ }^{4}$, Latha Palaniappan ${ }^{1,2 \ddagger}$, and \\ Francois Haddad ${ }^{1,2 \ddagger}$
}

${ }^{1}$ Division of Cardiovascular Medicine, Stanford University School of Medicine, Stanford, CA, USA; ${ }^{2}$ Stanford Cardiovascular Institute, 300 Pasteur Dr H2170, Stanford, CA 94305, USA; ${ }^{3}$ Stanford Sports Cardiology, Stanford University, Stanford, CA, USA; ${ }^{4}$ Research Unit Hypertension and Cardiovascular Epidemiology, Department of Cardiovascular Sciences, University of Leuven, Kapucijnenvoer 35 blok d - box 70013000 Leuven, Belgium; ${ }^{5}$ Department of Clinical Physiology, Linköping University, SE-581 83 Linköping, Sweden; ${ }^{6}$ Department of Medical and Health Sciences, Linköping University, SE-581 83 Linköping, Sweden; ${ }^{7}$ Department of Genetics, Stanford University School of Medicine, Stanford, CA, USA; ${ }^{8}$ Division of Cardiology, Veterans Affairs Palo Alto Healthcare System and Stanford University, 3801 Miranda Avenue, Palo Alto, CA 94304, USA; and ${ }^{9}$ Department of Medicine, Stanford Center for Clinical Research, 300 Pasteur Dr, Stanford, CA 94305, USA

Received 2 January 2020; editorial decision 20 March 2020; accepted 25 March 2020

Aims

Resting echocardiography is a valuable method for detecting subclinical heart failure (HF) in patients with diabetes mellitus (DM). However, few studies have assessed the incremental value of diastolic stress for detecting subclinical HF in this population.

Methods and results

\begin{abstract}
Asymptomatic patients with Type 2 DM were prospectively enrolled. Subclinical HF was assessed using systolic dysfunction (left ventricular longitudinal strain $<16 \%$ at rest and $<19 \%$ after exercise in absolute value), abnormal cardiac morphology, or diastolic dysfunction $\left(E / e^{\prime}>10\right)$. Metabolic equivalents (METs) were calculated using treadmill speed and grade, and functional capacity was assessed by percent-predicted METs (PPMETs). Among 161 patients studied (mean age of $59 \pm 11$ years and $57 \%$ male sex), subclinical HF was observed in $68 \%$ at rest and in $79 \%$ with exercise. Among characteristics, diastolic stress had the highest yield in improving detection of HF with $57 \%$ of abnormal cases after exercise and $45 \%$ at rest. Patients with revealed diastolic dysfunction during stress had significantly lower exercise capacity than patients with normal diastolic stress $(7.3 \pm 2.1$ vs. $8.8 \pm 2.5, P<0.001$ for peak METs and $91 \pm 30 \%$ vs. $105 \pm 30 \%, P=0.04$ for PPMETs). On multivariable modelling found that age (beta $=$ -0.33), male sex (beta $=0.21$ ), body mass index (beta $=-0.49$ ), and exercise $E / e^{\prime}>10$ (beta $=-0.17$ ) were independently associated with peak METs (combined $R^{2}=0.46$ ). A network correlation map revealed the connectivity of peak METs and diastolic properties as central features in patients with DM.
\end{abstract}

Conclusion Diastolic stress test improves the detection of subclinical HF in patients with diabetes mellitus.

Keywords diastolic stress • diabetic cardiomyopathy • early-stage heart failure 


\section{Introduction}

Detecting early-stage heart failure (HF) in patients with diabetes mellitus (DM) has been shown to identify patients at higher risk of poor cardiovascular outcome. For example, Dandamudi et al. ${ }^{1}$ reported that diastolic dysfunction predicts long-term mortality as well as development of $\mathrm{HF}$ in patients with DM. Other important features that help define early-stage HF include ventricular remodelling or hypertrophy as well as impaired left ventricular longitudinal strain (LVLS). Lam et al. ${ }^{2,3}$ demonstrated that left ventricular (LV) hypertrophy with increased relative wall thickness (RWT) is strongly associated with increased risk of HF with preserved ejection fraction (EF) and Liu et al. $^{4}$ reported that LVLS rather than LV ejection fraction (LVEF) is predictive of cardiovascular events in patients with Type 2 DM. Cauwenberghs et al. ${ }^{5}$ have also demonstrated the importance of subclinical cardiac remodelling, along with LV systolic and diastolic dysfunction in predicting cardiovascular events and these parameters were complementary to the atherosclerotic cardiovascular disease risk score.

While early-stage diabetic cardiomyopathy (DCM) has usually been defined based on resting studies, exercise testing could help further define subclinical HF by assessing contractile reserve and diastolic stress. Contractile reserve measures the ability of the ventricle to increase its systolic performance under stress (exercise or pharmacological), while diastolic stress assesses whether filling pressure increases during exercise. Previous studies have reported that patients with DM exhibit reduced contractile function during exercise, ${ }^{6}$ while other studies have demonstrated that overt LV diastolic dysfunction during exercise was associated with impaired functional capacity in patients with Type 2 DM. ${ }^{7,8}$ Recently, diastolic stress echocardiography has been proposed to uncover diastolic dysfunction in those patients who do not present diastolic dysfunction at rest. $^{9-11}$

To date, few studies have assessed the incremental value of exercise testing for the detection of early-stage HF in patients with DM. The first objective of our study was to compare ventricular remodelling and function at rest and with exercise between controls and participants with DM. Our second objective was to investigate whether diastolic stress improves the sensitivity of detecting subclinical HF. Finally, we investigated whether patients in whom diastolic dysfunction was revealed by exercise had lower exercise capacity than those with normal diastolic dysfunction.

\section{Methods}

\section{Study population}

We prospectively recruited patients with Type 2 DM participating in one of two physical activity clinical trials [Initiate and Maintain Physical Activity in Clinics (IMPACT) study (NCT02061579) ${ }^{12}$ or the Strength Training Regimen for Normal Weight Diabetics (STRONG-D) study (NCT02448498)]. ${ }^{13}$ We included subjects who agreed to participate in a cardiac phenotype substudy between September 2016 and November 2018, where they completed comprehensive resting as well as stress echocardiography testing at screening. Patients were excluded from the final analysis if a diagnosis of chronic kidney disease, neuropathy, diagnosed liver disease, or active malignancy was present. Furthermore, we included 25 subjects without DM for the purpose of comparison. This cohort consisted of subjects who participated in the $\mathrm{NIH}$ integrated Human Microbiome Project 2 (iHMP) as a self-reported healthy volunteer ${ }^{14}$ and agreed to participate in an exercise study. The absence of diabetes was determined by fasting plasma glucose (FPG) and $\mathrm{HbA} 1 \mathrm{C}$ levels measured within 3 months of the exercise date (FPG $<126 \mathrm{mg} / \mathrm{dL}$ and $\mathrm{HbA} 1 \mathrm{C}$ $<6.5 \%$ ). The study was approved by the Stanford Institutional Review Board and all participants gave written informed consent.

\section{Resting and exercise echocardiography}

Echocardiography was performed using commercially available echocardiographic systems (EPIQ 7C; Philips Medical Imaging, Eindhoven, the Netherlands), according to the American Society of Echocardiography guideline recommendations. ${ }^{15}$ Image analyses were performed on Xcelera workstations by trained cardiologists from the Biomarker and Imaging Core Laboratory at Stanford Cardiovascular Institute (Y.K. and T.N.). LVEF was calculated using Simpson's method. RWT was calculated as ( $2 *$ inferolateral wall thickness)/(LV internal dimension) and LV concentric remodelling was defined as RWT $>0.42 .{ }^{15} \mathrm{LV}$ mass was obtained using the linear method and LV hypertrophy was defined as LV mass index $\left(\right.$ LVMI) $>95 \mathrm{~g} / \mathrm{m}^{2}$ for women and LVMI $>115 \mathrm{~g} / \mathrm{m}^{2}$ for men. Transmitral pulsed-wave Doppler and tissue Doppler imaging were acquired from apical four-chamber view to obtain early $(E)$ and late $(A)$ diastolic flow velocity as well as systolic $\left(s^{\prime}\right)$ and early diastolic $\left(e^{\prime}\right)$ velocity of the mitral annulus at septal and lateral. E/e' ratio and $s^{\prime}$ were obtained by the average of the septal and lateral site. LV longitudinal strain (LVLS) was measured using Lagrangian strain by manual tracing from the apical views, computing the myocardial length in end-diastole $\left(L_{0}\right)$ and endsystole $\left(L_{1}\right)$ in the following formula: $100 \times\left(L_{1}-L_{0}\right) / L_{0}$ as described before. ${ }^{16}$ Since $L_{1}$ is smaller than $L_{0}$, LVLS is obtained as negative values, however, for consistency between ventricular measures, LVLS was presented in absolute value in this study. Left atrial (LA) volumes were obtained using biplane area-length method and LA strain was measured using Lagrangian strain by manual tracing as previously described. ${ }^{17} \mathrm{LA}$ reservoir strain was calculated with QRS onset as $100 \times\left(L_{\max }-L_{\min }\right) / L_{\text {min }}$ where $L_{\max }$ represents the maximum length and $L_{\min }$ represents the minimum length.

LV diastolic function was assessed by the combination of pulsed-wave Doppler examination of mitral inflow and tissue Doppler imaging of mitral annulus. Diastolic function was categorized according to the progression of diastolic dysfunction: normal $\left(0.75<E / A<1.5\right.$ and $\left.E / \mathrm{e}^{\prime}<10\right)$; mild, defined as impaired relaxation without evidence of increased filling pressures $\left(E / A \leq 0.75\right.$ and $\left.E / e^{\prime}<10\right)$; moderate, defined as impaired relaxation associated with moderate elevation of filling pressures or pseudonormal filling $\left(0.75<E / A<1.5\right.$ and $\left.E / e^{\prime} \geq 10\right)$, and severe, defined as advanced reduction in compliance or reversible or fixed restrictive filling (E/A $>1.5$ and $E / e^{\prime} \geq 10$ ) as previously described and validated. ${ }^{18,19}$

Subclinical HF at rest was evaluated by LV morphology, systolic and diastolic function. Abnormal morphology was present if patients presented LV concentric remodelling or LV hypertrophy. Systolic dysfunction was defined as LVLS in absolute value $<16 \% .{ }^{20}$ Diastolic dysfunction was defined by the presence of elevation of filling pressure (i.e. E/e' ratio $\geq 10)^{18,19}$ and LV end-diastolic pressure was estimated using the previously reported equation $[11.96+0.596 \times($ lateral E/e' $)] \cdot{ }^{21}$ We used LVEF, LVLS, and $s^{\prime}$ to assess contractile reserve and $E / e^{\prime}$ ratio to assess diastolic stress after exercise. The cut-offs of LVEF, LVLS and $s^{\prime}$ after exercise were determined as lower 5 th percentile of control subjects; LVEF 
as $68 \%, \mathrm{LVLS}$ as $19.0 \%$, and $\mathrm{s}^{\prime}$ as $7.3 \mathrm{~cm} / \mathrm{s}$. The same threshold at rest $\left(E / \mathrm{e}^{\prime}\right.$ ratio $\geq 10$ ) was used for defining diastolic dysfunction after exercise, as normal diastolic function allows adequate filling of the ventricles during rest and exercise without abnormal elevation of LV filling pressure. ${ }^{22,23}$

\section{Exercise stress test and functional capacity}

All patients performed exercise echocardiography using a symptomlimited treadmill ramp protocol. Exercise protocols were individualized according to the estimated exercise capacity of the participant using the Veterans Specific Activity Questionnaire. ${ }^{24}$ Peak metabolic equivalents of tasks (METs) were calculated using treadmill peak speed and grade. Predicted METs based on age and sex were calculated, using the Veterans Affairs cohort formula for men calculated as METs $=18-(0.15$ $\times$ age) and the St. James Take Heart Project formula for women as METs = $14.7-(0.13 \times$ age $) .{ }^{25-27}$ Percent-predicted METs $($ PPMETs $)$ was calculated as $100 \times($ METs achieved/age-gender predicted METs) to assess functional capacity; PPMETs $<85 \%$ was regarded as decreased functional capacity. ${ }^{27}$

\section{Statistical analysis}

Variables are presented as counts and percentages or mean and standard deviation. Categorical variables were compared using Pearson's $\chi^{2}$ test or Fisher's exact test, as appropriate. Normality of the continuous variables was confirmed with Shapiro-Wilk test. Comparison was performed using Student's or Welch $t$-test or Mann-Whitney $U$ test, as appropriate for two groups. One-way ANOVA or Kruskal-Wallis tests were used for comparison between three groups, and post hoc analysis was performed with Turkey-HSD multiple comparison tests, Games-Howell, or DunnBonferroni, as appropriate. Multivariable linear or logistic regression analysis was performed to detect the independent associates of peak METs or pPMETs $<85 \%{ }^{27}$ using the covariates as age, sex, body mass index (BMI), HbA1c, presence of hypertension, systolic blood pressure, medications including beta-blockers, calcium channel blockers, diuretics, and angiotensin-converting-enzyme inhibitors/angiotensin II receptor blockers, and the presence of subclinical HF, LA reservoir strain at rest, or impaired contractile reserve (LVLS in absolute value after exercise $<19 \%)$ and diastolic dysfunction after exercise (E/e' ratio after exercise $\geq 10$ ). Correlation network analysis was further performed to visualize the relationships between parameters with a threshold of $P$-values $<0.05$ for edges. Edges were coloured based on the direction of correlation between parameters and the thickness of the edges represented absolute value of Pearson's correlation $r$-value. $P$-values $<0.05$ were considered statistically significant. Analyses were performed using SPSS version 24 (SPSS Inc., Chicago, IL, USA).

\section{Results}

We prospectively enrolled 180 asymptomatic individuals with DM in the study (Figure 1). Among them, one participant was excluded due to exercise-induced ventricular tachycardia and two were excluded due to exercise-induced wall motion abnormalities. Furthermore, 16 patients were excluded due to technical difficulty in image acquisition at the end, a total of 161 individuals were included in the final analysis. There were no differences between all patients enrolled whole studies $(N=616)$ and 161 patients included in this analysis regarding age $(57.9 \pm 10.7$ vs. $59.2 \pm 10.7$ years, $P=0.17)$, sex ( $59 \%$ vs. $57 \%$ for male sex, $P=0.53)$, or HbA1c $(7.6 \pm 1.1 \%$ vs. $7.5 \pm 1.0 \%, P=0.55)$.

Participants with DM did not differ from controls in age $(P=0.74)$, sex $(P=0.96)$, or BMI $(P=0.86)$, while the prevalence of hypertension was higher in patients with DM $(P<0.001$, Table 1$)$.

\section{Resting echocardiography}

As shown in Table 1, patients with DM had higher LVMI, RWT, impaired LVLS, as well as higher E/e' ratio. LA volume index did not

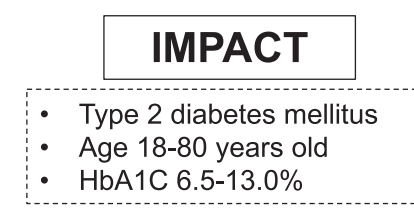

\section{STRONG-D}

- Type 2 diabetes mellitus

- Age 30-75 years old

- $\mathrm{HbA} 1 \mathrm{C} 6.5-13.0 \%$

- BMI $18.5-25 \mathrm{~kg} / \mathrm{m}^{2}$

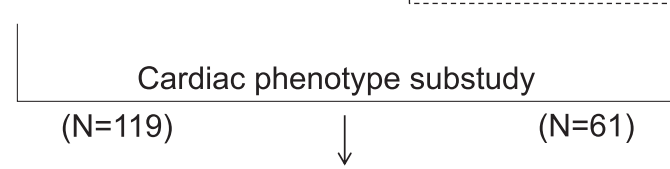

Resting and stress echocardiographic assessment

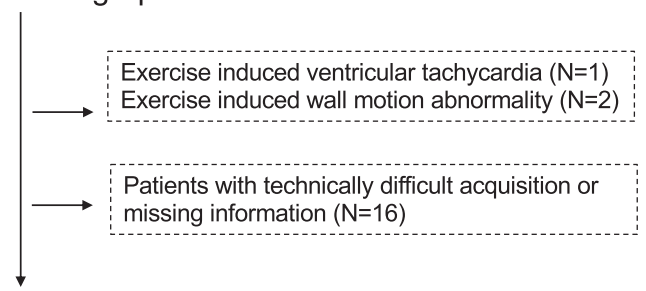

Patients included in the final analysis

$(\mathrm{N}=161)$

Figure I Flow chart of the study. 
Table I Baseline characteristics

\begin{tabular}{|c|c|c|c|}
\hline & $\begin{array}{l}\text { Controls } \\
(N=25)\end{array}$ & $\begin{array}{l}\text { Diabetes } \\
(N=161)\end{array}$ & $P$-value \\
\hline Age (years) & $59.6 \pm 6.1$ & $59.2 \pm 10.7$ & 0.74 \\
\hline Male sex, $n(\%)$ & $14(56)$ & $91(57)$ & 0.96 \\
\hline Body mass index $\left(\mathrm{kg} / \mathrm{m}^{2}\right)$ & $29.6 \pm 5.2$ & $29.4 \pm 6.9$ & 0.86 \\
\hline Hypertension, $n(\%)$ & $4(16)$ & $116(72)$ & $<0.001$ \\
\hline Heart rate (bpm) & $63 \pm 12$ & $80 \pm 12$ & $<0.001$ \\
\hline Systolic blood pressure (mmHg) & $126 \pm 12$ & $136 \pm 16$ & $<0.001$ \\
\hline Diastolic blood pressure $(\mathrm{mmHg})$ & $76 \pm 9$ & $81 \pm 11$ & 0.03 \\
\hline HbA1c (\%) & $5.5 \pm 0.3$ & $7.5 \pm 1.0$ & $<0.001$ \\
\hline \multicolumn{4}{|l|}{ Medication } \\
\hline Beta-blockers & $0(0)$ & $14(9)$ & 0.13 \\
\hline Calcium channel blockers & $0(0)$ & $15(9)$ & 0.11 \\
\hline Diuretics & $0(0)$ & $22(14)$ & 0.049 \\
\hline ACE-I/ARBs & $0(0)$ & $73(45)$ & $<0.001$ \\
\hline \multicolumn{4}{|l|}{ Resting echocardiographic parameters } \\
\hline Left ventricular dimension $(\mathrm{cm})$ & $4.8 \pm 0.4$ & $4.6 \pm 0.5$ & 0.07 \\
\hline Relative wall thickness & $0.29 \pm 0.04$ & $0.39 \pm 0.07$ & $<0.001$ \\
\hline $\mathrm{LV}$ mass index $\left(\mathrm{g} / \mathrm{m}^{2}\right)$ & $62.0 \pm 9.6$ & $70.4 \pm 17.2$ & 0.001 \\
\hline LVEF (\%) & $61.6 \pm 3.9$ & $64.8 \pm 4.8$ & $<0.001$ \\
\hline LVLS (\%) & $19.2 \pm 1.2$ & $16.7 \pm 1.6$ & $<0.001$ \\
\hline $\mathrm{s}^{\prime}(\mathrm{cm} / \mathrm{s})$ & $7.7 \pm 1.0$ & $7.5 \pm 1.5$ & 0.35 \\
\hline$E / e^{\prime}$ & $7.7 \pm 2.0$ & $10.3 \pm 2.9$ & $<0.001$ \\
\hline LA volume index $\left(\mathrm{mL} / \mathrm{m}^{2}\right)$ & $24.8 \pm 6.3$ & $26.4 \pm 7.0$ & 0.21 \\
\hline LA reservoir strain (\%) & $36.7 \pm 5.4$ & $30.1 \pm 9.7$ & $<0.001$ \\
\hline
\end{tabular}

ACE-I, angiotensin-converting-enzyme inhibitors; ARB, angiotensin II receptor blockers; LVEF, left ventricular ejection fraction; LVLS, left ventricular longitudinal strain; METs, metabolic equivalents.

differ between patients with DM and controls; however, LA reservoir strain was significantly lower in patients with DM.

Of all patients, 44 patients (27\%) presented with abnormal morphology, 49 patients (31\%) presented with impaired LVLS, and 72 patients (45\%) presented with diastolic dysfunction. Subclinical HF based on one criterion was present in 65 (40\%), by two criteria in 35 (22\%) and by three criteria in $10(6 \%)$, while 51 patients (32\%) did not present any abnormalities.

\section{Exercise stress test}

One hundred thirty-six patients with DM (87\%) reached peak heart rate $(H R) \geq 85 \%$ of their age-predicted maximum $H R$ compared with 24 controls $(96 \%)(P=0.20)$. Peak METs $(P=0.20)$ and PPMETs $(P=0.21)$ were comparable between patients with $D M$ and controls; however, more patients with DM tended to have reduced exercise capacity $(P=0.06)$ (Table 2$)$.

\section{Diastolic stress and contractile reserve in patients with DM}

During exercise, LVLS and $s^{\prime}$ were slightly but significantly impaired in patients with DM while LVEF was comparable (Table 2). For the detection of subclinical HF, diastolic stress provided the greatest yield; diastolic dysfunction was observed in 72 participants (45\%) at rest and in 92 (57\%) after exercise (Figure 2A). Figure 2B shows the change

\begin{tabular}{|c|c|c|c|}
\hline & $\begin{array}{l}\text { Controls } \\
(N=25)\end{array}$ & $\begin{array}{l}\text { Diabetes } \\
(N=161)\end{array}$ & $P$-value \\
\hline Peak heart rate (bpm) & $155 \pm 12$ & $154 \pm 19$ & 0.80 \\
\hline Peak systolic blood pressure (mmHg) & $164 \pm 20$ & $186 \pm 22$ & $<0.001$ \\
\hline Peak diastolic blood pressure $(\mathrm{mmHg})$ & $80 \pm 11$ & $83 \pm 12$ & 0.37 \\
\hline Peak METs & $8.7 \pm 2.4$ & $7.9 \pm 2.4$ & 0.20 \\
\hline Percent-predicted METs (\%) & $106 \pm 20$ & $100 \pm 29$ & 0.21 \\
\hline$<85 \%$ ppMETs, $n(\%)$ & $4(16)$ & $52(32)$ & 0.06 \\
\hline \multicolumn{4}{|c|}{ Immediately after exercise echocardiographic parameters } \\
\hline $\operatorname{LVEF}(\%)$ & $73.9 \pm 3.7$ & $74.2 \pm 4.3$ & 0.74 \\
\hline $\operatorname{LVLS}(\%)$ & $22.1 \pm 1.6$ & $21.0 \pm 2.2$ & 0.002 \\
\hline$s^{\prime}(\mathrm{cm} / \mathrm{s})$ & $11.6 \pm 2.6$ & $10.4 \pm 2.3$ & 0.01 \\
\hline$E / e^{\prime}$ & $8.0 \pm 2.1$ & $10.8 \pm 2.9$ & $<0.001$ \\
\hline \multicolumn{4}{|l|}{ Change from rest to after exercise } \\
\hline Absolute change in LVEF (\%) & $12.3 \pm 4.4$ & $9.3 \pm 4.4$ & 0.003 \\
\hline Relative change in LVEF (\%) & $20.3 \pm 8.3$ & $14.8 \pm 7.5$ & 0.001 \\
\hline Absolute change in LVLS (\%) & $3.0 \pm 1.5$ & $4.3 \pm 1.9$ & 0.001 \\
\hline Relative change in LVLS (\%) & $15.7 \pm 8.2$ & $26.6 \pm 13.1$ & $<0.001$ \\
\hline Absolute change in $\mathrm{s}^{\prime}(\mathrm{cm} / \mathrm{s})$ & $4.0 \pm 2.2$ & $2.9 \pm 1.9$ & 0.02 \\
\hline Relative change in $s^{\prime}(\%)$ & $51.9 \pm 28.6$ & $41.1 \pm 27.8$ & 0.05 \\
\hline
\end{tabular}

LVEF, left ventricular ejection fraction; LVLS, left ventricular longitudinal strain; METs, metabolic equivalents.

of $E / e^{\prime}$ from at rest to after exercise and identifies a group of revealed diastolic dysfunction. Patients were classified into three groups; no diastolic dysfunction both at rest and after exercise $(N=63$, Normal), diastolic dysfunction at rest $(N=72$, Resting DD) and no diastolic dysfunction at rest but diastolic dysfunction revealed after exercise ( $N=26$, Revealed DD). In contrast, the yield for detection of subclinical HF was not observed for LVEF, LVLS, or s' (Figure 2C$E)$. Figure $2 F$ summarizes the abnormal ratio in those parameters at rest and after exercise. Adding diastolic stress to resting parameters increased the number of patients with subclinical cardiac abnormalities especially patients with more than two abnormal features compared with adding contractile reserve (Figure 3).

\section{Comparison between groups of diastolic stress}

The comparative features according to the groups classified by diastolic function are summarized in Table 3. When compared with the Normal group, patients in either the Resting DD or the Revealed DD group had lower peak METs (Figure 4A). The Resting DD group which also had lower peak METs were the oldest and with a higher proportion of females (Table 3).

\section{Peak exercise capacity and early heart failure profiles}

As shown in the heatmap of Figure 4B, peak METs had moderate associations with age, male sex, and BMI. Peak METs also correlated 


\section{A. E/e'}

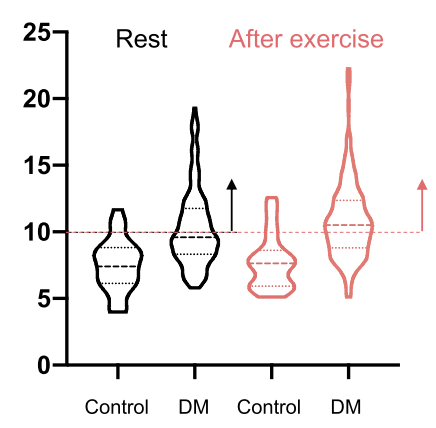

D. Absolute LVLS (\%)

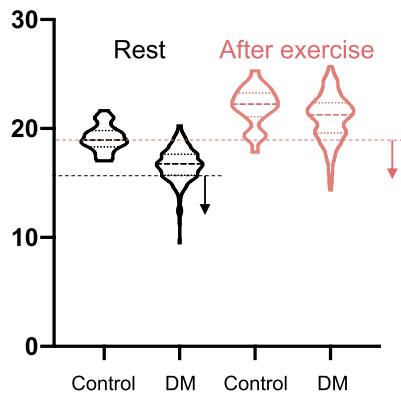

B. E/e' change

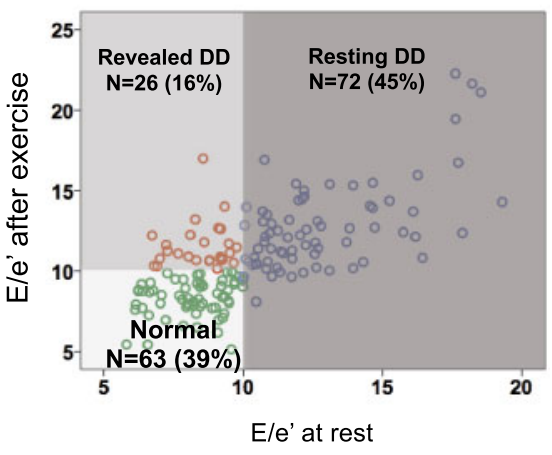

E. $\mathrm{s}^{\prime}(\mathrm{cm} / \mathrm{s})$

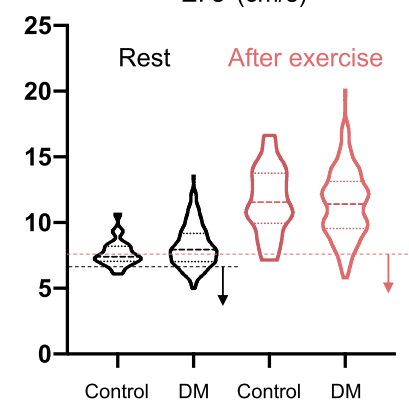

C. LVEF (\%)

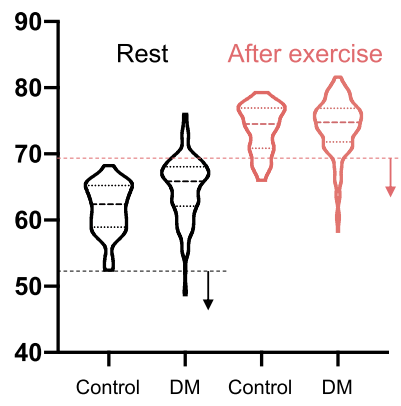

F. Ratio of patients with abnormal values

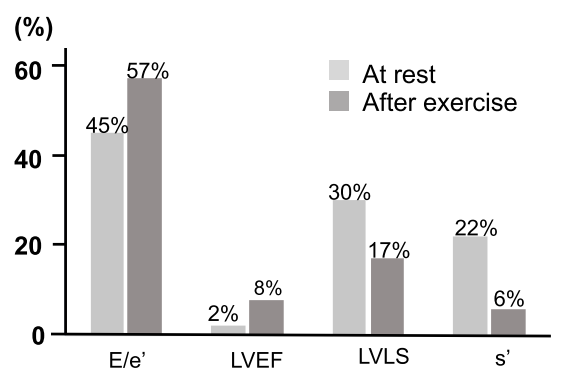

Figure 2 Comparison of systolic and diastolic function at rest and after exercise between controls and patients with DM. (A) The violin plots of resting (black) and after exercise (red) in E/e' ratio. (B) The change of E/e' ratio from rest to after exercises. The green circle represents patients in Normal group, the blue circle represents resting diastolic dysfunction group, and the red circle represents patients in revealed diastolic dysfunction group. ( $C-E)$ The violin plots resting (black) and after exercise (red) in LVEF (C), LVLS (D), and s' $(E)$. (F) The ratio of patients with abnormal diastolic and systolic function at rest and after exercise. LVEF, left ventricular ejection fraction; LVLS, left ventricular longitudinal strain.

\section{A Resting parameters}

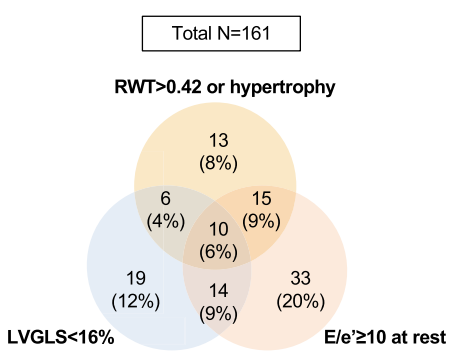

None: $51(32 \%)$
B Adding diastolic stress to rest

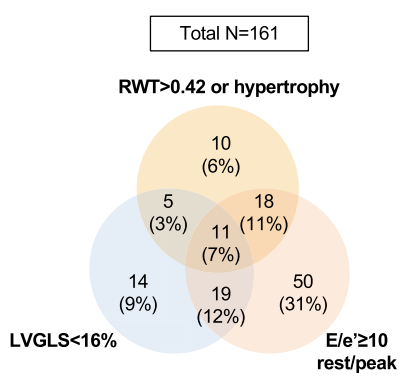

None: $34(21 \%)$

\section{Ratio of subclinical cardiac abnormalities}

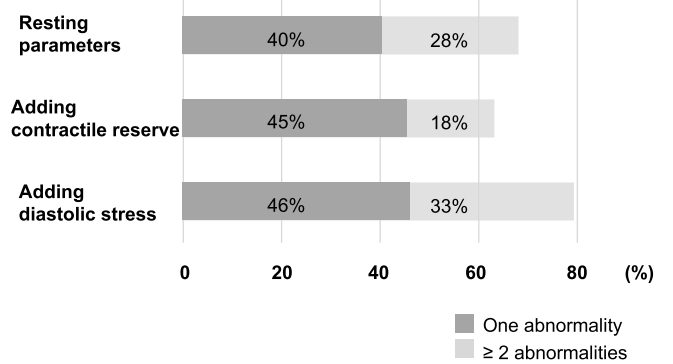

Figure 3 Prevalence of subclinical HF. ( $A$ and $B$ ) Venn diagrams demonstrating the overlap between patients with abnormal morphology change (RWT $>0.42$ or LV hypertrophy), LV systolic and diastolic dysfunction by only resting echocardiographic assessment (A) as well as adding diastolic stress assessment (B). (C) Prevalence of subclinical HF evaluated by resting echocardiographic assessment, adding contractile reserve, and adding diastolic stress. Adding diastolic stress helped to detect more patients with $\geq 2$ cardiac abnormalities.

most strongly with $s^{\prime}$ and $E / e^{\prime}$ among resting echocardiographic parameters and with $s^{\prime}$ and $E / e^{\prime}$ immediately after exercise. On multivariable analysis, age (beta $=-0.34)$, male sex $($ beta $=0.21)$, BMI (beta $=\times 0.49)$, and peak $E / e^{\prime} \geq 10$ (beta $=\times 0.17$ ) were independently associated with peak METs $\left(R^{2}=0.46\right)$ (Figure $4 C$ ). However, medications including beta-blockers, HbA1c and reduced contractile reserve were not significantly associated with peak METs or reduced exercise capacity. The correlation network (Figure 4D) 
Table 3 Baseline characteristics and echocardiographic parameters according to diastolic function

\begin{tabular}{|c|c|c|c|c|}
\hline & Normal $(N=63)$ & Resting DD $(N=72)$ & Revealed DD $(N=26)$ & $P$-value \\
\hline Age (years) & $56.7 \pm 10.2$ & $61.5 \pm 10.5 *$ & $58.8 \pm 11.5$ & 0.03 \\
\hline Male sex, $n(\%)$ & $42(67)$ & $32(44)$ & $17(65)$ & 0.02 \\
\hline Body mass index $(\mathrm{kg} / \mathrm{m} 2)$ & $28.8 \pm 6.8$ & $29.9 \pm 6.9$ & $27.8 \pm 5.2$ & 0.28 \\
\hline Hypertension, $n$ (\%) & $45(71)$ & $54(75)$ & $17(65)$ & 0.64 \\
\hline $\mathrm{HbA1c}(\%)$ & $7.6 \pm 1.2$ & $7.5 \pm 0.9$ & $7.6 \pm 1.1$ & 0.85 \\
\hline \multicolumn{5}{|l|}{ Resting echocardiographic parameters } \\
\hline Relative wall thickness & $0.39(0.35-0.42)$ & $0.39 \pm 0.07$ & $0.37 \pm 0.07$ & 0.46 \\
\hline $\mathrm{LV}$ mass index $(\mathrm{g} / \mathrm{m} 2)$ & $68.0 \pm 14.7$ & $72.3 \pm 18.7$ & $71.3 \pm 18.0$ & 0.49 \\
\hline LVEF (\%) & $64.2 \pm 4.6$ & $65.3 \pm 5.0$ & $64.5 \pm 4.8$ & 0.19 \\
\hline LVLS (\%) & $16.8 \pm 1.4$ & $16.6 \pm 1.7$ & $16.9 \pm 1.4$ & 0.41 \\
\hline$s^{\prime}(\mathrm{cm} / \mathrm{s})$ & $8.2 \pm 1.3$ & $6.7 \pm 1.2^{*}$ & $7.9 \pm 1.4 \#$ & $<0.001$ \\
\hline$E / e^{\prime}$ & $8.2 \pm 1.2$ & $12.8 \pm 2.4^{*}$ & $8.5 \pm 1.0 \#$ & $<0.001$ \\
\hline $\mathrm{LA}$ volume index $(\mathrm{mL} / \mathrm{m} 2)$ & $26.1 \pm 7.3$ & $26.7 \pm 6.6$ & $26.1 \pm 7.8$ & 0.91 \\
\hline LA reservoir strain (\%) & $31.7 \pm 8.8$ & $28.1 \pm 10.5$ & $31.2 \pm 9.4$ & 0.06 \\
\hline \multicolumn{5}{|l|}{ Peak exercise } \\
\hline Heart rate (bpm) & $162 \pm 16$ & $149 \pm 20^{*}$ & $152 \pm 19$ & 0.001 \\
\hline Systolic blood pressure $(\mathrm{mmHg})$ & $192 \pm 23$ & $185 \pm 22$ & $185 \pm 22$ & 0.39 \\
\hline Peak METs & $9.0 \pm 2.6$ & $7.3 \pm 2.3^{*}$ & $7.3 \pm 2.1 *$ & $<0.001$ \\
\hline Percent-predicted METs (\%) & $105 \pm 28$ & $98 \pm 29$ & $91 \pm 30$ & 0.13 \\
\hline$<85 \%$ ppMETs, $n(\%)$ & $15(26)$ & $25(37)$ & $12(52)$ & 0.07 \\
\hline \multicolumn{5}{|c|}{ Immediately after exercise echocardiographic parameters } \\
\hline $\operatorname{LVEF}(\%)$ & $74.7 \pm 3.9$ & $73.7 \pm 4.6$ & $73.6 \pm 3.9$ & 0.27 \\
\hline LVLS in absolute value (\%) & $21.2 \pm 2.0$ & $20.5 \pm 2.4$ & $21.7 \pm 1.8 \#$ & 0.03 \\
\hline$s^{\prime}(\mathrm{cm} / \mathrm{s})$ & $11.6 \pm 2.3$ & $9.3 \pm 2.0^{*}$ & $10.5 \pm 1.8 \#$ & $<0.001$ \\
\hline$E / e^{\prime}$ & $8.3 \pm 1.1$ & $12.8 \pm 2.8^{*}$ & $11.6 \pm 1.5^{*}$ & $<0.001$ \\
\hline
\end{tabular}

highlights the central connectivity of peak METs and diastolic function characteristics as well as the correlation between parameters in patients with DM.

\section{Discussion}

The main finding of our study was that diastolic stress is incremental to resting echocardiographic parameters in defining subclinical HF in patients with DM. Furthermore, diastolic stress could delineate the mechanisms of exercise limitations in patients with DM.

DCM is a major risk factor for adverse events in patients with DM and detecting subclinical DCM can offer the opportunity for early intervention. However, resting parameters may not fully capture abnormalities in asymptomatic patients with DM. Recently, there is growing evidence that diastolic stress provides important diagnostic findings that can be of value in the management of patients presenting with dyspnoea of unclear aetiology or due to HF. For example, Obokata et al. ${ }^{11}$ examined the value of diastolic stress echocardiography in patients with HF with preserved EF (HFpEF) and they observed that even in the absence of E/e' elevation at rest, patients displayed elevated filling pressures exclusively during exercise stress. In those patients, diastolic stress provided additional information that could clarify the diagnosis of early-stage HFpEF. The importance of diastolic stress test is also emphasized in a recently published consensus document from the European Society of Cardiology on the diagnosis of HFpEF, which recommends diastolic stress test as an additional workup in the absence of elevated LV filling pressures at rest. $^{28}$

One of the important findings of our study is that diastolic dysfunction was revealed by exercise in $16 \%$ of these patients. Given that diastolic dysfunction has been regarded as the first manifestation of DCM,29-31 diastolic stress may further increase its diagnostic yields. In contrast, we observed that contractile reserve assessed by LVEF or LVLS was mostly maintained during exercise despite impaired LVLS at rest in a majority of our population. This may be partly because our population was composed asymptomatic patients with likely still preserved contractile reserve.

Consistent with previous studies which have demonstrated an association between reduced exercise capacity and increased filling pressure during exercise, ${ }^{7,8,11}$ our study showed an inverse association between diastolic function and exercise capacity. Impaired LV diastolic function increases pulmonary artery wedge pressure, which in sequence could alter total lung perfusion and the distribution of pulmonary blood flow, and thus could induce relative pulmonary congestion. This may become a restricting factor when a greater 
A Distribution of peak METs and $\mathrm{ppETs}$
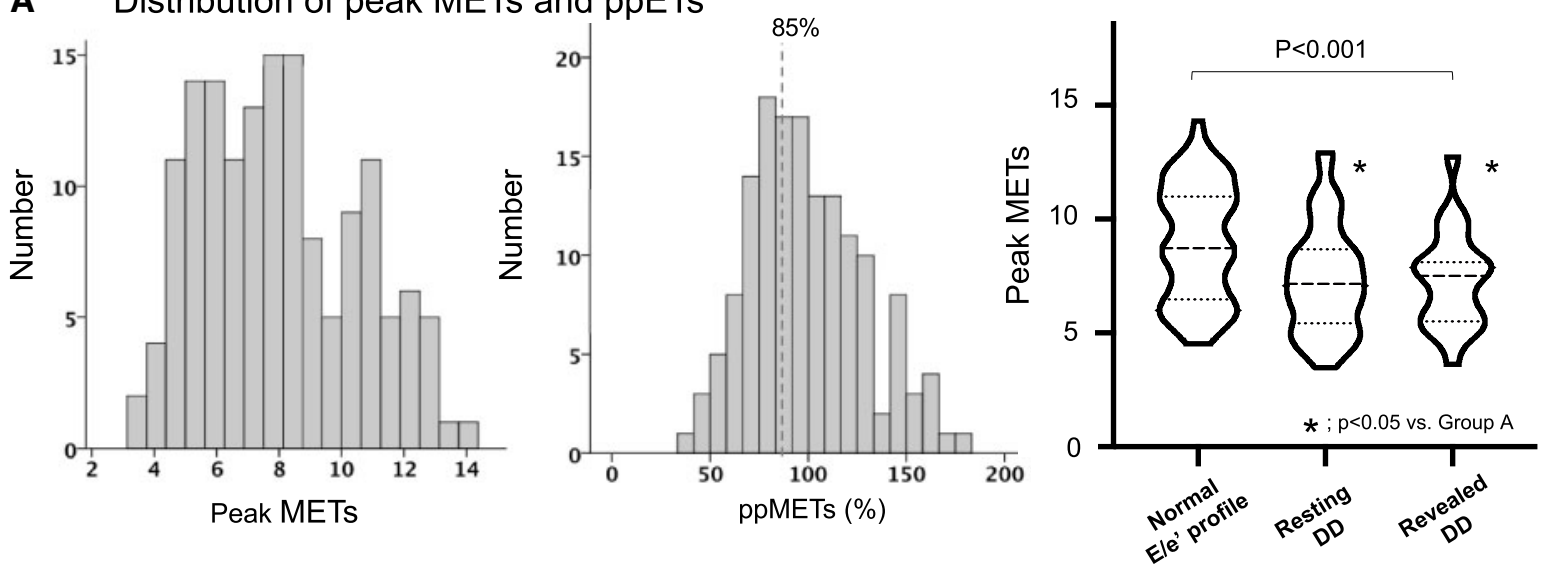

B Correlation of exercise capacity

D Network between parameters
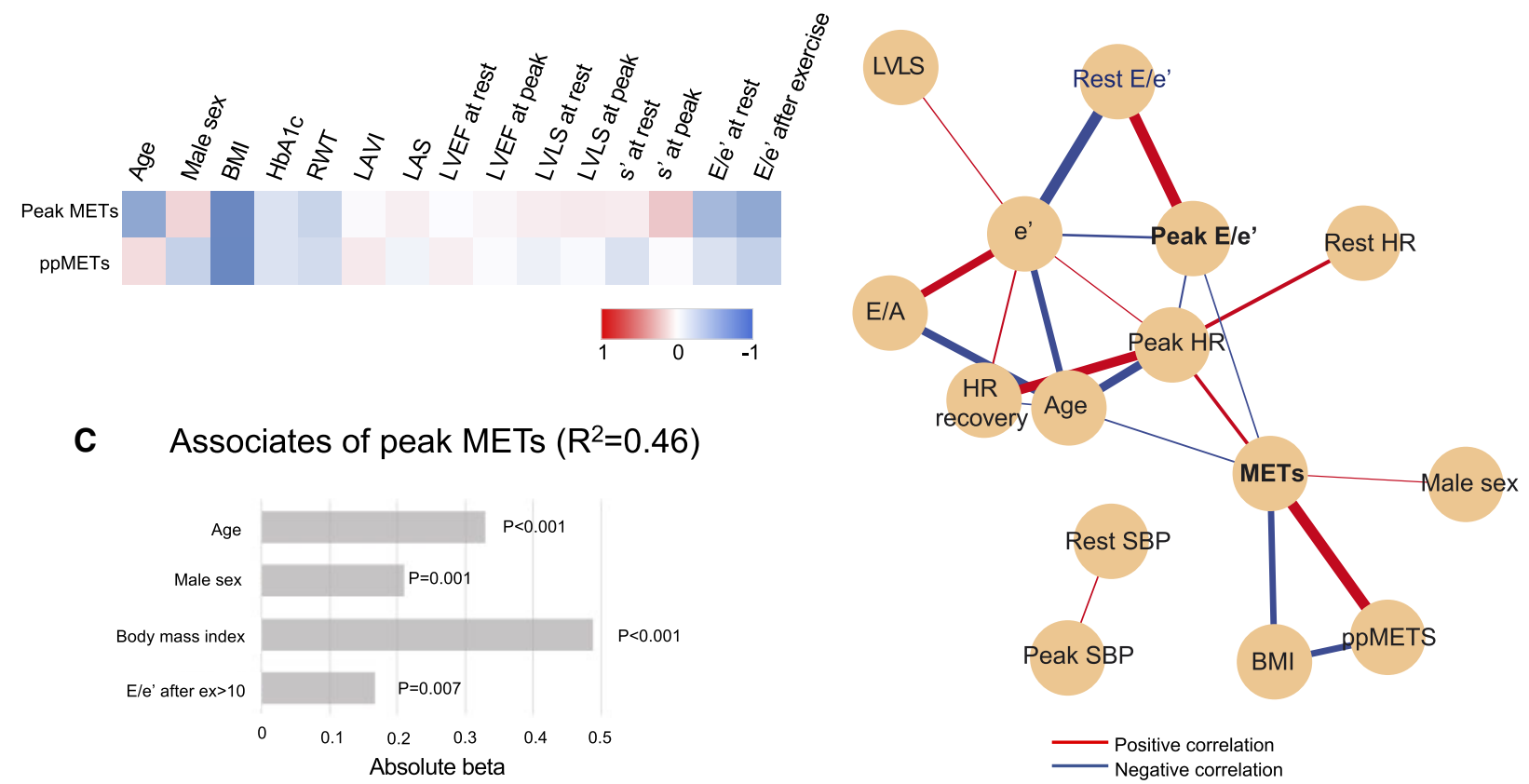

Figure 4 (A) Distribution of peak METs and pPMETs. (B) A heatmap showing relationship between exercise capacity and characteristics as well as cardiac function at rest and after exercise. The exercise capacity was more related to cardiac function after exercise compared to that at rest. (C) Independent associates of peak METs. (D) Correlation network. BMI, body mass index; HR, heart rate; LAS, left atrial strain; LVEF, left ventricular ejection fraction; LVLS, left ventricular longitudinal strain; METs, metabolic equivalents; RWT, relative wall thickness; SBP, systolic blood pressure; $\mathrm{LAVI}$, left atrial volume index.

exercise performance is required but not limit activities of daily living. This may explain the response in patients with DM in the current study, where $E / e^{\prime}$ at rest was normal but elevated after exercise. Patients with DM often complain of reduced exercise capacity and it has been reported that exercise performance is abnormal in these patients. $^{32,33}$ In addition, diastolic dysfunction after exercise was more strongly related to reduced exercise capacity compared to that at rest, highlighting the importance of assessing diastolic stress even when diastolic function is maintained at rest.
We used the criteria to diagnose diastolic dysfunction using E/e' ratio with lower threshold than that in the current American Society of Echocardiography and the European Association of Cardiovascular Imaging (ASE/EACVI) guidelines. ${ }^{34}$ Since E/e' is often used in assessing filling pressure change for diastolic stress, ${ }^{11}$ we focused on the combination of pulsed-wave Doppler of mitral inflow and tissue Doppler imaging of mitral annulus to assess diastolic function, where we used the threshold of E/e 10 instead of 14 , which is supported by the study by Redfield et al. ${ }^{18}$ Given that the early detection to risk stratify in patients with DM potentially leads to tailored 
intense preventive strategies, we used more sensitive criteria to diagnose diastolic dysfunction in DCM in this study. Indeed, a lot more patients were classified as Normal using E/e' threshold of $14 \mathrm{com}$ pared with $E / e^{\prime}$ of 10 . Further studies are warranted to investigate if more sensitive criteria would help predict outcome in patients with DM.

LA reservoir strain was significantly reduced in asymptomatic patients with DM even in the absence of LA enlargement, which is similar to a previous study. ${ }^{35}$ Since DM is one of the independent risk factors for the development of atrial fibrillation, ${ }^{36}$ the reduction of $L A$ reservoir strain regardless of $L A$ enlargement might be helpful for risk stratification of the development of atrial fibrillation. In addition, E/e' after exercise has been reported to predict the development of new-onset atrial fibrillation regardless of LA enlargement. ${ }^{37}$ Larger prospective studies might be important to perform which investigate if the combination of reduced LA reservoir strain and impaired diastolic stress would develop atrial fibrillation. Furthermore, given that the reduced LA function might be caused by LV diastolic dysfunction, this provides some insights on potential considerations for trial design to determine target parameters for screening or during follow-up.

Our study suggests that diastolic stress test was of value to detect subclinical cardiac abnormalities and provided additional information to distinguish subjects with early pathology. Consequently, diastolic stress might yield better inclusion criteria in future prospective, interventional study design.

There are several limitations in our study. First, the number of the population was relatively small because patients were selected prospectively for participation in exercise programs, and thus not representative of a general population and they may have been more fit and motivated compared to general patients with DM. Second, the number of controls was relatively small compared to patients with DM in this study. Third, there is currently no obvious threshold for LVLS after exercise, therefore, we used a value obtained from our control subjects. The 2016 ASE/EACVI recommendations use E/e', $e^{\prime}$ velocity, TR velocity and left atrial volume index to evaluate the presence of diastolic dysfunction, however, optimal TR velocity was available in 99 patients (61\%) at rest and in 19 patients (12\%) after exercise in our population. Also, to simplify the criteria to define diastolic dysfunction for a screening, we used the combination of E/A and $E / e^{\prime}$ ratio to diagnose. Further studies are warranted for more precise assessment of contractile reserve or diastolic stress to relate outcome. Finally, with the revised and lower diagnostic criteria for hypertension and based on the fact that hypertension often co-exists with DM, we used a broader definition of DCM including hypertension in the absence of ischaemic heart disease in this study. This explains why we preferentially used the term subclinical HF rather than DCM in the article. In our population, hypertension related with LV morphology change but did not with other cardiac functions.

\section{Conclusion}

Diastolic stress has incremental value to resting parameters in defining subclinical HF in patients with DM and is associated with reduced exercise capacity. In addition, diastolic stress has a higher sensitivity than contractile reserve to detect subclinical HF, after excluding subjects with stress-induced wall motion abnormalities. This could thus have potential value in diagnosis and risk stratification of patients with DM, especially in those with exercise intolerance.

\section{Funding}

This work was supported by the Stanford Diabetes Research Center (P30DK116074), the National Institutes of Health under award number R18DK09639405 and R01DK08137110, as well as the grand from Philips Royal Precision Medicine Initiative.

Conflict of interest: None declared.

\section{References}

1. Dandamudi S, Slusser J, Mahoney DW, Redfield MM, Rodeheffer RJ, Chen $\mathrm{HH}$. The prevalence of diabetic cardiomyopathy: a population-based study in Olmsted County, Minnesota. J Card Fail 2014;20:304-9.

2. Lam CSP, Lyass A, Kraigher-Krainer E, Massaro JM, Lee DS, Ho JE et al. Cardiac dysfunction and noncardiac dysfunction as precursors of heart failure with reduced and preserved ejection fraction in the community. Circulation 2011;124: 24-30.

3. Lam CSP, Roger VL, Rodeheffer RJ, Bursi F, Borlaug BA, Ommen SR et al. Cardiac structure and ventricular-vascular function in persons with heart failure and preserved ejection fraction from Olmsted County, Minnesota. Circulation 2007;115:1982-90.

4. Liu J-H, Chen Y, Yuen M, Zhen Z, Chan C-S, Lam K-L et al. Incremental prognostic value of global longitudinal strain in patients with type 2 diabetes mellitus. Cardiovasc Diabetol 2016;15:22.

5. Cauwenberghs N, Hedman K, Kobayashi Y, Vanassche T, Haddad F, Kuznetsova T. The 2013 ACC/AHA risk score and subclinical cardiac remodeling and dysfunction: complementary in cardiovascular disease prediction. Int J Cardiol 2019; 297:67-74.

6. Ha J-W, Lee H-C, Kang E-S, Ahn C-M, Kim J-M, Ahn J-A et al. Abnormal left ventricular longitudinal functional reserve in patients with diabetes mellitus: implication for detecting subclinical myocardial dysfunction using exercise tissue Doppler echocardiography. Heart 2007;93:1571-6.

7. Poirier P, Garneau C, Bogaty P, Nadeau A, Marois L, Brochu C et al. Impact of left ventricular diastolic dysfunction on maximal treadmill performance in normotensive subjects with well-controlled type 2 diabetes mellitus. Am J Cardiol 2000;85:473-7.

8. Ozkan H, Akdemir S, Tiryakioglu S, Ari H, Bozat T. The evaluation of type 2 diabetes mellitus related changes in diastolic dysfunction during exercise using conventional and tissue doppler echocardiography. Cardiol Res 2015;6:346-51.

9. Burgess MI, Jenkins C, Sharman JE, Marwick TH. Diastolic stress echocardiography: hemodynamic validation and clinical significance of estimation of ventricular filling pressure with exercise. J Am Coll Cardiol 2006;47:1891-900.

10. Tartière-Kesri L, Tartière J-M, Logeart D, Beauvais F, Solal AC. Increased proximal arterial stiffness and cardiac response with moderate exercise in patients with heart failure and preserved ejection fraction. J Am Coll Cardiol 2012;59: 455-61.

11. Obokata M, Kane GC, Reddy YNV, Olson TP, Melenovsky V, Borlaug BA. Role of diastolic stress testing in the evaluation for heart failure with preserved ejection fraction: a simultaneous invasive-echocardiographic study. Circulation 2017; 135:825-38.

12. Faroqi L, Wong M, Bonde S, Wong CW, Walai K, West W et al. Evaluating the clinical implementation of structured exercise: a randomized controlled trial among non-insulin dependent type II diabetics. Contemp Clin Trials 2018;74: 25-31.

13. Faroqi L, Bonde S, Goni DT, Wong CW, Wong M, Walai K et al. STRONG-D: strength training regimen for normal weight diabetics: rationale and design. Contemp Clin Trials 2019;78:101-6.

14. Zhou W, Sailani MR, Contrepois K, Zhou Y, Ahadi S, Leopold SR et al. Longitudinal multi-omics of host-microbe dynamics in prediabetes. Nature 2019; 569:663-71

15. Lang RM, Badano LP, Mor-Avi V, Afilalo J, Armstrong A, Ernande L et al. Recommendations for cardiac chamber quantification by echocardiography in adults: an update from the American Society of Echocardiography and the European Association of Cardiovascular Imaging. J Am Soc Echocardiogr 2015;28: 1-39.e14.

16. Kobayashi Y, Ariyama M, Kobayashi Y, Giraldeau G, Fleischman D, Kozelj M et al. Comparison of left ventricular manual versus automated derived longitudinal strain: implications for clinical practice and research. Int J Cardiovasc Imaging 2016; 32:429-37.

17. Kobayashi Y, Moneghetti KJ, Boralkar K, Amsallem M, Tuzovic M, Liang D et al. Challenging the complementarity of different metrics of left atrial function: insight 
from a cardiomyopathy-based study. Eur Heart J Cardiovasc Imaging 2017;18: 1153-62.

18. Redfield MM, Jacobsen SJ, Burnett JC, Jr, Mahoney DW, Bailey KR, Rodeheffer RJ. Burden of systolic and diastolic ventricular dysfunction in the community: appreciating the scope of the heart failure epidemic. JAMA 2003; 289:194-202.

19. Ommen SR, Nishimura RA, Appleton CP, Miller FA, Oh JK, Redfield MM et al. Clinical utility of Doppler echocardiography and tissue Doppler imaging in the estimation of left ventricular filling pressures: a comparative simultaneous Doppler-catheterization study. Circulation 2000;102:1788-94.

20. Yingchoncharoen T, Agarwal S, Popović ZB, Marwick TH. Normal ranges of left ventricular strain: a meta-analysis. J Am Soc Echocardiogr 2013;26:185-91.

21. Ohtani T, Mohammed SF, Yamamoto K, Dunlay SM, Weston SA, Sakata Y et al. Diastolic stiffness as assessed by diastolic wall strain is associated with adverse remodelling and poor outcomes in heart failure with preserved ejection fraction. Eur Heart J 2012;33:1742-9.

22. Kitzman DW, Higginbotham MB, Cobb FR, Sheikh KH, Sullivan MJ. Exercise intolerance in patients with heart failure and preserved left ventricular systolic function: failure of the Frank-Starling mechanism. I Am Coll Cardiol 1991;17: 1065-72.

23. Ha JW, Lulic F, Bailey KR, Pellikka PA, Seward JB, Tajik AJ et al. Effects of treadmill exercise on mitral inflow and annular velocities in healthy adults. Am J Cardiol 2003;91:114-5.

24. Myers J, Buchanan N, Walsh D, Kraemer M, McAuley P, Hamilton-Wessler M et al. Comparison of the ramp versus standard exercise protocols. J Am Coll Cardiol 1991;17:1334-42.

25. Morris CK, Myers J, Froelicher VF, Kawaguchi T, Ueshima K, Hideg A. Nomogram based on metabolic equivalents and age for assessing aerobic exercise capacity in men. J Am Coll Cardiol 1993;22:175-82.

26. Gulati M, Pandey DK, Arnsdorf MF, Lauderdale DS, Thisted RA, Wicklund RH et al. Exercise capacity and the risk of death in women. Circulation 2003;108: 1554-9.

27. Kim ESH, Ishwaran H, Blackstone E, Lauer MS. External prognostic validations and comparisons of age- and gender-adjusted exercise capacity predictions. J Am Coll Cardiol 2007;50:1867-75.
28. Pieske B, Tschöpe C, Boer R. D, Fraser AG, Anker SD, Donal E et al. How to diagnose heart failure with preserved ejection fraction: the HFA-PEFF diagnostic algorithm: a consensus recommendation from the Heart Failure Association (HFA) of the European Society of Cardiology (ESC). Eur Heart J 2019;40: 3297-317.

29. Seneviratne BI. Diabetic cardiomyopathy: the preclinical phase. $\mathrm{Br}$ Med J 1977;1: 1444-6.

30. Ahmed SS, Jaferi GA, Narang RM, Regan TJ. Preclinical abnormality of left ventricular function in diabetes mellitus. Am Heart J 1975;89:153-8.

31. Raev DC. Which left ventricular function is impaired earlier in the evolution of diabetic cardiomyopathy? An echocardiographic study of young type I diabetic patients. Diabetes Care 1994;17:633-9.

32. Regensteiner JG, Sippel J, McFarling ET, Wolfel EE, Hiatt WR. Effects of noninsulin-dependent diabetes on oxygen consumption during treadmill exercise. Med Sci Sports Exerc 1995;27:875-81.

33. Estacio RO, Regensteiner JG, Wolfel EE, Jeffers B, Dickenson M, Schrier RW The association between diabetic complications and exercise capacity in NIDDM patients. Diabetes Care 1998;21:291-5.

34. Nagueh SF, Smiseth OA, Appleton CP, Byrd BF 3rd, Dokainish H, Edvardsen T et al. Recommendations for the evaluation of left ventricular diastolic function by echocardiography: an update from the American Society of Echocardiography and the European Association of Cardiovascular Imaging. Eur Heart I Cardiovasc Imaging 2016;17:1321-60.

35. Cameli M, Mandoli GE, Lisi E, Ibrahim A, Incampo E, Buccoliero G et al. Left atrial, ventricular and atrio-ventricular strain in patients with subclinical heart dysfunction. Int J Cardiovasc Imaging 2019;35:249-58.

36. Movahed M-R, Hashemzadeh M, Jamal MM. Diabetes mellitus is a strong, independent risk for atrial fibrillation and flutter in addition to other cardiovascular disease. Int J Cardiol 2005;105:315-8.

37. Takagi T, Takagi A, Yoshikawa J. Elevated left ventricular filling pressure estimated by $E / E^{\prime}$ ratio after exercise predicts development of new-onset atrial fibrillation independently of left atrial enlargement among elderly patients without obvious myocardial ischemia. J Cardiol 2014;63:128-33. 